\title{
Modeling of ionizing radiation effect on static and dynamic behavior of vertical cavity surface emitting lasers
}

\author{
Sh.M. Eladl, K.A. Sharshar \\ Radiation Eng. Dept., NCRRT, \\ Atomic Energy Authority, P. O. Box 29, 3 Ahmed Elzomor str., \\ 8th sector, Nasr city, Cairo, Egypt.
}

\begin{abstract}
In this work, the effect of ionizing radiation on static and dynamic behavior of Vertical Cavity Surface Emitting Laser (VCSEL) was investigated numerically. First, the model of dynamic behavior before irradiation has been analyzed based on Convolution Theorem. Second, all interesting ionizing radiation sensitive factors are compared with their corresponding post irradiation factors. The convolution theorem is applied to get features of dynamic behavior. All interesting parameters have been outlined. The effect of resonance frequency and damping parameters have been studied. The results show that static and dynamic response of these devices are dramatically deteriorated due to irradiation flux. The device gradually changes its mode of operation from lasing mode to LED mode by exhibiting weak oscillation of the output and fast damping with the increase of ionizing radiation. This type of model can be used for high data bit rate in multimode optical fiber network.
\end{abstract}

Keywords: vertical cavity surface emitting laser, ionizing radiation, LED.

Manuscript received 24.05.17; revised version received 28.09.17; accepted for publication 07.12.17; published online 07.12.17.

\section{Introduction}

The vertical-cavity surface-emitting laser (VCSEL) is a new class of semiconductor laser that is fabricated monolithically [1]. This class of emitters can be considered as an important type that promises the optical fiber communication systems revolution, since it has several advantages over edge-emitting lasers [2].

Emission of coherent light through VCSEL takes place perpendicular to the integrated layers of its structure, while the edge-emitting laser diodes emits coherent light parallel to the integrated layers [3]. The appearance of output light emerging vertically through the layers comes from the schematic diagram showing the planes in a horizontal manner [4].

VCSELs can operate at wavelengths near infrared (IR) spectrum like 850 and $1300 \mathrm{~nm}$. The longest visible wavelength is at approximately $770 \mathrm{~nm}$. Recently [2], a novel design of $1530 \mathrm{~nm}$ long-wavelength device with high direct modulation speed was developed for high speed optical communication systems and optical interconnection network. VCSELs are always fabricated from materials like gallium arsenide (GaAs), aluminum gallium arsenide (AlGaAs), and indium gallium arsenide nitride (InGaAsN) [5, 6].

A great interest of VCSELs is focused due to their several advantages over other types of laser sources. They are cheaper for manufacturing, easier for testing, and have high efficiency. In addition, the coherent output light energy is produced by a less electrical current. A narrow spectral width is emitted by VCSELs, also, the obtained beam is nearly circular as compared with the other light sources. This and other make an efficient coupling between VCSEL and optical fiber [7-9]. 
The goal of this paper is to analyze the effects of ionizing radiation damage on the transient response of VCSEL. The analytical model is based on introducing the irradiation identities such as the threshold current, relaxation oscillation frequency, and damping factor into the obtained characteristic equations concerning the dynamic behavior.

\section{Theoretical analysis}

\subsection{Structure and operation of the device}

The construction of VCSEL is mainly different from other lasers. VCSEL is composed of a vertical cavity developed by epitaxial layers and contains a Distributed Bragg Reflectors (DBR) on the top and bottom of the active region. Fig. 1 shows a model of VCSEL [8]. The DBR mirrors are highly reflective mirrors, with the reflectivity higher than $99.9 \%$. The two DBRs in the VCSEL are oppositely doped ( $n$-DBR and $p$-DBR). The active layer of laser is placed between the upper and lower DBRs and contains several quantum wells. The active region receives current through guiding layers to emit intense lasing light.

\subsection{Dynamic behavior}

To understand dynamic behavior of VCSEL, it is required to start with the transfer function characteristic equation described in Ref. [7, 8] as follows
$M(\omega)=\frac{P(\omega)}{I(\omega)}=\frac{h \nu}{q} \eta_{d} \frac{\omega_{r}^{2}}{\omega_{r}^{2}-\omega^{2}+j \omega \gamma}$,

where $\omega_{r}$ is the relaxation oscillation and $\gamma$ is the damping parameter described as

$\omega_{r}=\sqrt{\frac{1}{2 \pi} \frac{v_{g} \eta_{i} a}{q V_{m}}\left(I-I_{t h}\right)}$,

$\gamma=\frac{1}{\tau}+K \omega_{r}^{2}$

Here, $q$ is the unit charge, $\eta_{i}$ - injection efficiency, $\tau-$ carrier lifetime, $v_{g}$ - photon group velocity, $g$ - gain coefficient, $V_{m}$ - cavity volume, $K$ - damping factor and $\left(I-I_{t h}\right)$ - injection current above the threshold.

Assuming a unit step input injection current and following the convolution theorem described in [10], one can get

$$
P(t)=I(t) U(t) * M(t)
$$

where * denotes the convolution between $I(t) U(t)$ and $M(t)$.

Applying the convolution theorem in [10], one can get

$$
P(t)=I(t) U(t) * M(t)=\int_{0}^{t} U(t-\mu) * M(\mu) d \mu
$$

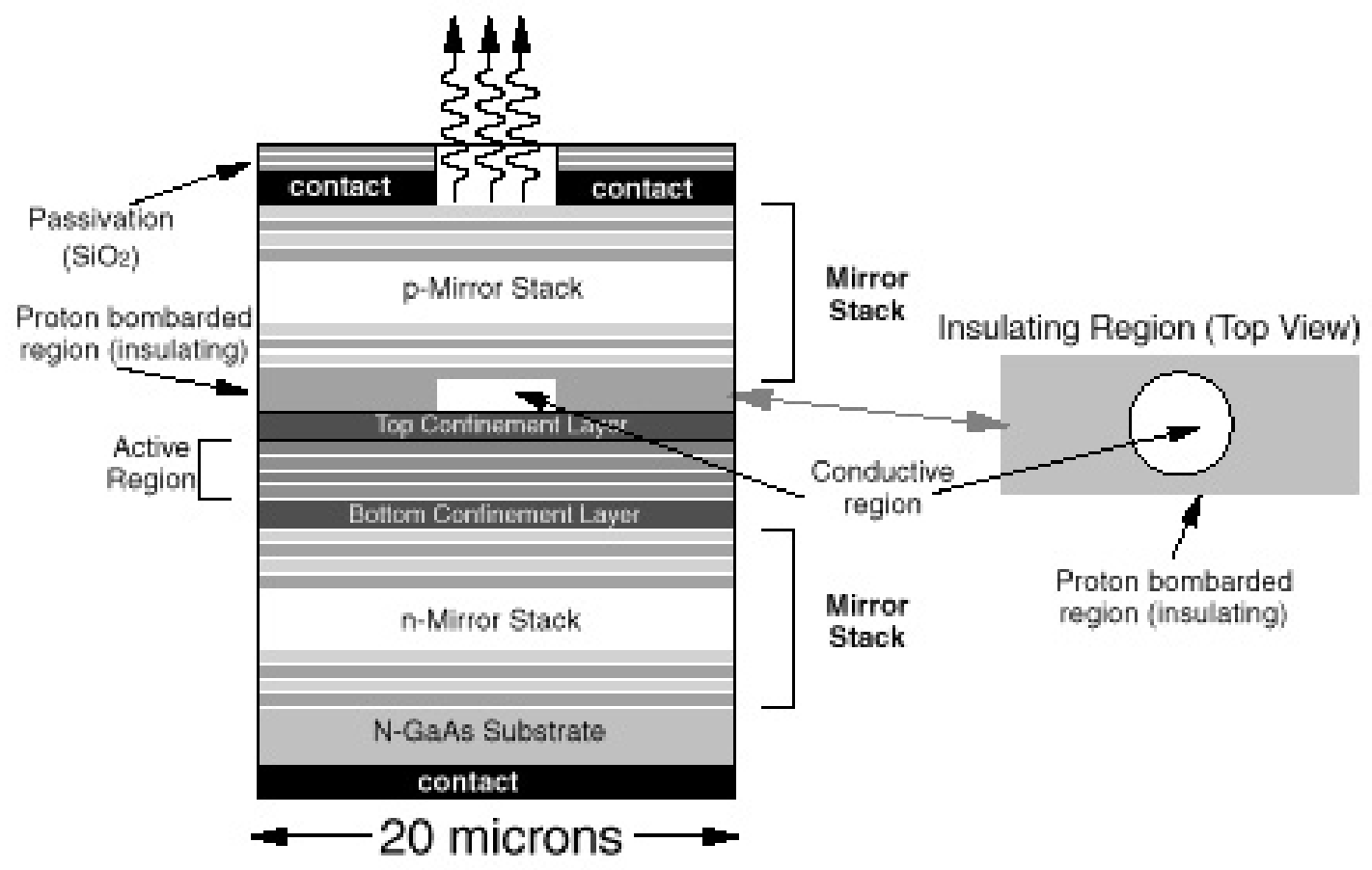

Fig. 1. Schematic structure of vertical cavity surface emitting laser (VCSEL) [8]. 
Here, $M(\mu)$ is the time domain of the transfer function $M(\omega)$ at instant $\mu$.

Then,

$$
P(t)=\int_{0}^{t} \frac{h v}{q} \eta_{d} \omega_{r} e^{-\gamma \mu} \sin \left(\omega_{r} t\right) d \mu .
$$

In the above expressions, $I(t) U(t)$ is the unit step input current equal to unity, and $U(t-\mu)$ is the delayed one, the above integration can be simplified as

$$
\begin{aligned}
& P(t)=\left(\frac{h v}{q}\right) \eta_{d} \times \\
& \times\left\{1-e^{-\gamma t}\left[\cos \left(\omega_{r} t\right)-\frac{\gamma^{2}}{\omega_{\mathrm{r}}^{2}} \cos \left(\omega_{r} t\right)+\frac{\gamma^{2}}{\omega_{\mathrm{r}}^{2}} \sin \left(\omega_{r} t\right)\right]\right\} .
\end{aligned}
$$

For $\omega_{r}>\gamma$, which corresponds to the lasing mode of the laser diode, one can obtain

$$
P(t)=\frac{h v}{q} \eta_{d}\left\{1-\cos \left(\omega_{r} t\right) e^{-\gamma t}\right\}
$$

Setting $\omega_{r}=0$ in Eq. (8), which corresponds to the LED mode of the laser diode, the output power is expressed as

$$
P(t)=\frac{h v}{q} \eta_{d}\left\{1-e^{-\gamma t}\right\} .
$$

\subsection{Ionizing radiation effect}

The output power of a laser diode is directly proportional to the lifetime of the excess minority carriers. The minority carrier lifetime damage can be described by [11]

$$
\frac{\tau_{0}}{\tau}=1+\tau_{0} K \Phi=1+F .
$$

Here, $\tau_{0}$ describes the preirradiation minority carrier lifetime, $\tau$ is the post-irradiation minority carrier lifetime, $K$ is a damage constant in $\mathrm{cm}^{2} / \mathrm{s}$, and $\Phi$ is the fluence of ionizing radiation in $\mathrm{cm}^{-2}$. If the irradiation fluence concerning neutrons, the unit is neutrons $/ \mathrm{cm}^{-2}$, while for gamma irradiation photons, the unit is photons $/ \mathrm{cm}^{2}$. As regard VCSEL, the included parameters within the characteristic equations that exhibit a change by ionizing radiation are $\omega_{r}, \gamma$, and $I_{t h}$. These factors are function of $F$, the threshold current include the parameter $\tau_{0}$ and can be written as [11]

$$
I_{t h}=\frac{q V n_{t h}}{\tau_{0}}
$$

After ionizing radiation, the above expression yields to $[11,12]$

$$
I_{t h}=\frac{q V n_{t h}}{\tau}=\frac{q V n_{t h}}{\tau_{0}}\left(1+\tau_{0} K \Phi\right)=\frac{q V n_{t h}}{\tau_{0}}(1+F),
$$

where $\mathrm{q}$ concerns the electron charge, $\mathrm{V}$ is the active region volume, nth - carrier density at threshold. From the above equation, the threshold current increases due to radiation and is proportional to $\mathrm{F}$. The parameter $\mathrm{K}$ depends on the type of radiation and the material structure. This parameter is a measure of the number of non-radiative centers.

\section{Results and discussions}

The device parameters used in the following calculations are the same as those used in Ref. [7], $\omega_{r}=10^{10} \mathrm{~Hz}$, $\gamma=10^{9} \mathrm{~Hz}$, the input injected current is assumed to be a unit step function in time. The dynamic response of the output optical power of VCSEL at different values of $F$ is shown in Fig. 2. The parameter $F$ is a measure of ionizing radiation value where it increases with increasing the irradiation flux. The generated figure is obtained for VCSEL that operates in the threshold mode. It can be seen that the output power of the device is decreased with the increase of $F$. Also, the rise time required for arriving to final state is decreased with the increase of $F$.

Dynamic behavior of VCSEL for different $F$ values is shown in Fig. 3. In this case, the device operates in the lasing mode, where $\omega_{r}=5 \mathrm{GHz}$. It is shown that when $F$ is increased, it leads to an increase of the damping factor and threshold current as well as to a decrement in relaxation oscillation $\omega_{r}$. As a result of that, the overshoot value is decreased. Also, the settling time is increased with the reduction of $F$. With the continuous increase of $F$, the VCSEL changes its mode of operation from the lasing one to the LED mode as in Fig. 3.

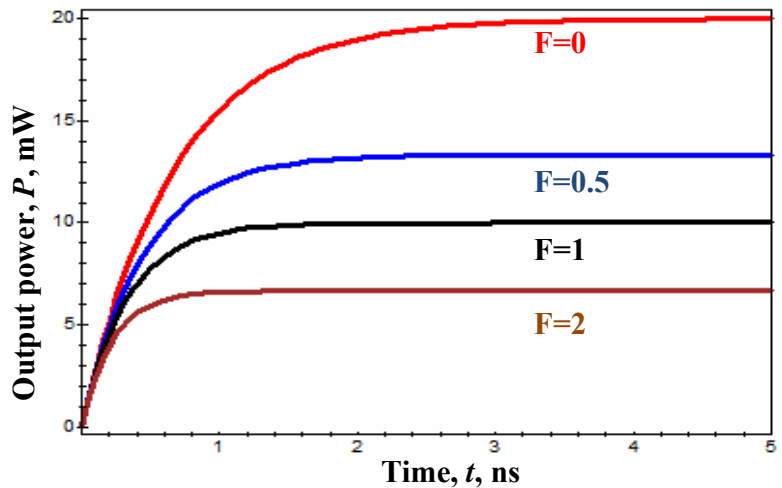

Fig. 2. Transient behavior of VCSEL at different values of $F$ in the LED mode $\left(\omega_{r}=0\right)$. 


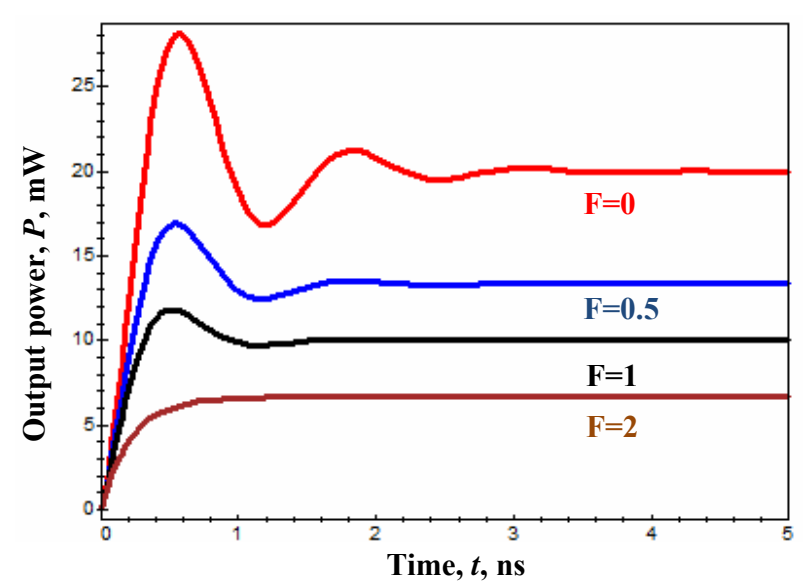

Fig. 3. Transient behavior of VCSEL at different values of $F$ and $\omega_{r}=5 \mathrm{GHz}$.

Fig. 4 shows dynamic behavior of VCSEL for different values of $F$. It is clear that the rise time is reduced with reducing $F$. As regards to the relation between the rise time and eye opening scheme, a fast rise time will contribute to larger vertical eye opening at high data rates, since the VCSEL can respond faster to the applied signal. Moreover, the signal amplitude is increased with reduced $F$ as a result of higher slope efficiency at the bias point, again contributing to a larger vertical eye opening.

The defect results by radiation damage to the VCSEL causes an increase in the non-radiative recombination rate and, hence, little amount of carriers would diffuse into the active region, and the laser could return faster to its steady-state condition. As regards to the threshold current, reduction of the minority carrier lifetime in the VCSEL layers could therefore lead to the increase in the threshold current after experienced to ionizing radiation as in Fig. 5.

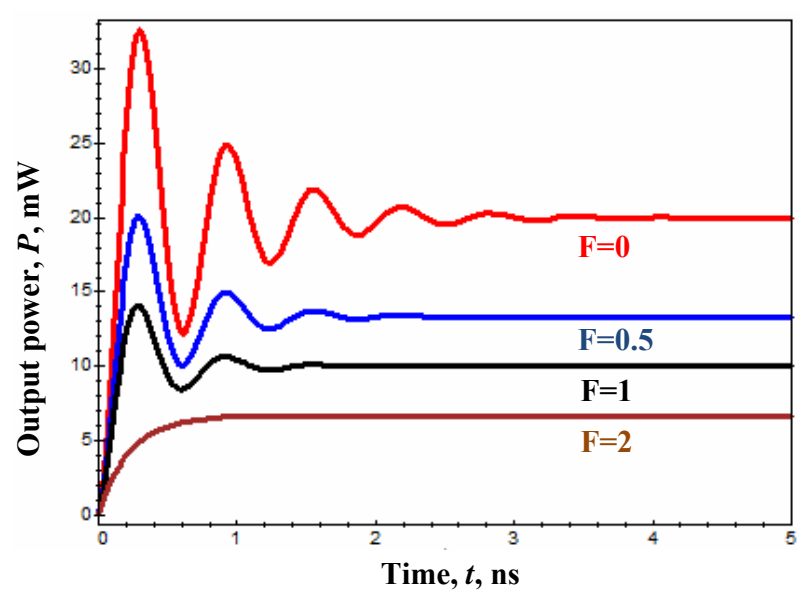

Fig. 4. Transient behavior of VCSEL at different values of $F$ and $\omega_{r}=10 \mathrm{GHz}$.

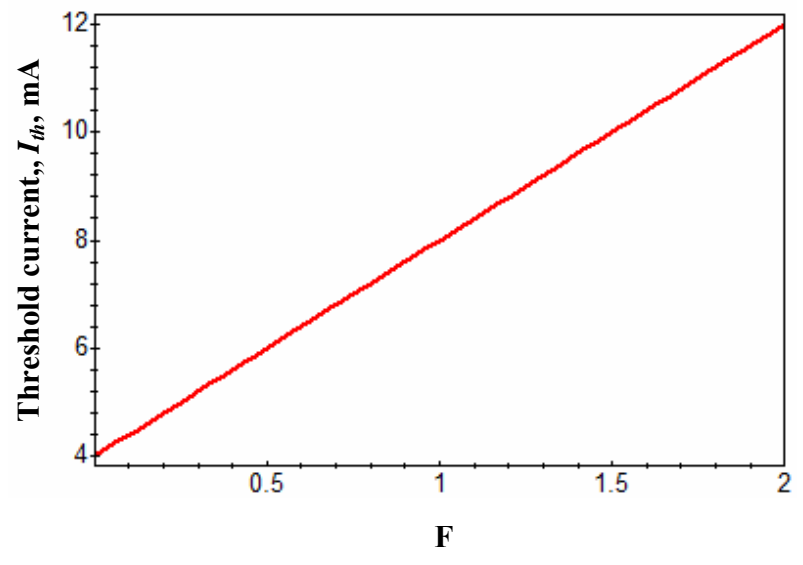

Fig. 5. Variation of threshold current versus $F$ value.

Fig. 6 shows the relaxation oscillation frequency $\left(\omega_{r}\right)$ dependence upon the injected current for the different values of $F$. The relaxation oscillation frequency depends on the current above threshold, which decreases with the increase of $F$ according to the latter figure, so the relaxation oscillation frequency yields to decrease with the increase of $F$.

\section{Conclusions}

The effect of ionizing radiation on static and dynamic behavior of vertical cavity surface emitting laser has been analyzed in this paper. The model is based on the use of convolution theorem to get dynamic behavior. The dynamic response is strongly affected by the ionizing irradiation flux. The ionizing radiation gradually changes the mode of operation of VCSEL from the laser mode to the LED one. This type of model can be used for the high data bit rate for the multimode optical fiber network.

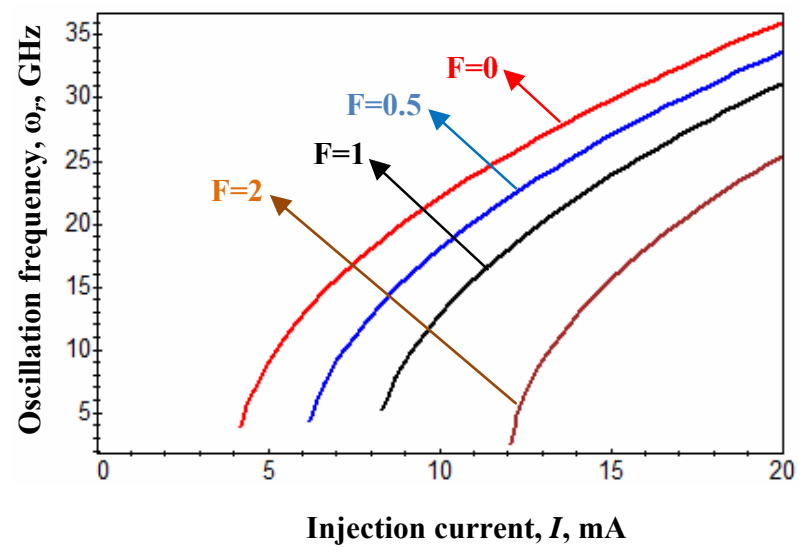

Fig. 6. Variation of oscillation frequency versus injection current at different values of $F$. 


\section{References}

1. Iga K. Vertical-cavity surface-emitting laser: Its conception and evolution. Jpn. J. Appl. Phys. 2008. 47, No. 1R. P. 1-10.

2. Malacarne A., Sorianello V., Daly A., Kögel B., Ortsiefer M., Neumeyr C., Romagnoli M. and Bogoni A. Performance analysis of $40-\mathrm{Gb} / \mathrm{s}$ transmission based on directly modulated highspeed 1530-nm VCSEL. IEEE Photon. Technol. Lett. 2016. 28, No. 16. P. 1735-1738.

3. Koyama F. VCSEL integration for silicon photonics. Proc. $3^{\text {rd }}$ IEEE Int. Conf. Group IV Photon. 2006. P. 194-196.

4. Aalto T., Harjanne M., Ylinen et al. Multiwavelength transceiver integration on SOI for highperformance computing system applications. Proc. SPIE. 2015. 9368. 93680B-1(10 p.).

5. Kuchta D.M., Schow C.L., Rylyakov A.V. et al. A 71-Gb/s NRZ modulated 850-nm VCSEL-based optical link. IEEE Photon. Technol. Lett. 2015. 27, No. 6. P. 577-580.

6. Al-Qazwini Z., Zhou J., and Kim H. 1.5- $\mu \mathrm{m}$ 10$\mathrm{Gb} / \mathrm{s}$ VCSEL link for optical access applications.
IEEE Photon. Technol. Lett. 2013. 25, No. 22. P. 2160-2163.

7. Hamad W., Wanckel S. and Hofmann W. Smallsignal analysis of ultra-high-speed multi-mode VCSELs. IEEE J. Quantum Electron. 2016. 52, No. 7. 2400311 (11 p.).

8. Raj M., Monge M., and Emami A. A modelling and nonlinear equalization technique for a $20 \mathrm{~Gb} / \mathrm{s} 0.77$ $\mathrm{pJ} / \mathrm{b}$ VCSEL transmitter in $32 \mathrm{~nm}$ SOI CMOS. IEEE J. Solid-State Circuits. 2016. 51, No. 8. P. 1734-1743.

9. Hofmann W. and Bimberg D. VCSEL-based light sources - scalability challenges for VCSEL-based multi-100-Gb/s systems. IEEE Photon. J. 2012. 4, No. 5. P. $1831-1843$.

10. Mishra R., Saxena R., and Jain R. Application of FRFT convolution theorem in filtering. Intern. $J$. Information Eng. 2013. 3. P. 71-75.

11. Barnes C.E. and Wiczer J.J. Radiation effects in optoelectronic devices. Sandia National Lab., SAND-84-0771, 1984.

12. Johnston A. Radiation effects in optoelectronic devices. IEEE Trans. Nucl. Sci. 2013. 60, No. 3. P. 2054-2073. 